Accepted for publication by GPS Solutions, doi: 10.1007/s10291-014-0384-6,

http://link.springer.com/article/10.1007\%2Fs10291-014-0384-6, ( ) Springer 2014

\title{
Multipath Mitigation and NLOS Detection using Vector Tracking in Urban
}

\section{Environments}

\author{
Li-Ta Hsu ${ }^{1}$, Shau-Shiun Jan ${ }^{1}$, Paul D Groves ${ }^{2}$, and Nobuaki Kubo ${ }^{3}$ \\ ${ }^{1}$ Department of Aeronautics and Astronautics, National Cheng Kung University, Taiwan \\ ${ }^{2}$ Faculty of Engineering, University College London, UK \\ ${ }^{3}$ Tokyo University of Marine Science and Technology, Japan
}

\begin{abstract}
Multipath interference and non-line-of-sight (NLOS) reception are major error sources when using global navigation satellite systems (GNSS) in urban environments. A promising approach to minimize the effect of multipath interference and aid NLOS detection is vector tracking. Therefore the objective of this research is to assess vector tracking in a dense urban environment to determine its effect on multipath interference and NLOS reception. Here, a vector delay lock loop (VDLL) is implemented using an adaptive extended Kalman filter (EKF). This replaces the individual code-tracking loops and navigation filter but retains conventional carrier frequency tracking. The positioning and tracking performance of the conventional and vector tracking implementations with and without a strobe correlator are compared using intermediate frequency (IF) signals recorded in the Koto-Ku district of urban canyon Tokyo city environment. Both static and dynamic tests were performed. It is shown that vector tracking reduces the root mean square positioning error by about $30 \%$ compared to an equivalent conventional receiver in urban environments and is capable of detecting long-delay NLOS reception for a GPS receiver without any external aiding.
\end{abstract}

\section{Introduction}

Multipath interference and non-line of sight (NLOS) reception are major sources of error for 
global navigation satellite systems (GNSS) in an urban environment (Misra and Enge 2011; Groves 2013). Buildings and vehicles obstruct, reflect, and diffract the signals. The pseudorange errors caused by the multipath interference can be reduced significantly through carefully designed antenna, such as antenna siting (McGraw et al. 2004), choke-ring antenna design (Braasch 1996). Receiver-based multipath mitigation techniques use modified discriminator designs, often with additional correlators, to improve the resolution of the signal correlation process (Dierendonck et al. 1992; Bhuiyan and Lohan 2010). This reduces the pseudorange multipath error when the receiver's precorrelation bandwidth is sufficient (Ward et al. 2006a). However, this is not always the case for a consumer receiver. Furthermore, these receiver-based techniques have no effect on NLOS reception (Groves 2013). New approaches to multipath and NLOS mitigation are therefore needed. A dual-polarization antenna can be used to detect NLOS reception, but is only suited to professional applications because of the additional hardware required (Jiang and Groves 2012). Consistency checking can also be used to identify both NLOS and multipath-contaminated signals, but is currently unreliable in dense urban environments (Groves and Jiang 2013). A review of multipath and NLOS mitigation techniques may be found in (Groves et al. 2013).

A promising approach to minimize the effect of multipath interference is vector tracking (Copps et al. 1980; Spilker 1996; Lashley et al. 2009). This replaces the delay lock loop (DLL) used for code tracking with an extended Kalman filter (EKF) that both tracks the GNSS signals and calculates the user position. Some implementations retain carrier tracking loops, while others incorporate carrier frequency tracking within the vector tracking algorithm. By combining the tracking and positioning tasks, vector tracking can use the user motion determined from the stronger GNSS signals to predict the code phase and maintain tracking of the weaker signals. Thus, vector tracking is more robust against signal interference and attenuation. This has been demonstrated under a range of conditions (Pany et 
al. 2005; Bhattacharyya and Gebre-Egziabher 2010; Lashley and Bevly 2011; Kanwal et al. 2010) and open source vector tracking algorithms are now available (Zhao and Akos 2011). Vector tracking has also been extended to incorporate carrier phase tracking (Petovello and Lachapelle 2006).

Vector tracking could also mitigate the effects of multipath interference and NLOS reception. Preliminary results using data recorded at the Tzu-Chang campus of National Cheng Kung University in Taiwan have demonstrated the potential of this technique (Hsu et al. 2013; Hsu 2013). Vector tracking is therefore assessed in a dense urban environment subject to high levels of multipath interference and NLOS reception. Results are presented using data collected in central Tokyo.

Because multipath interference has greater impact on the code than on the carrier, a vector delay lock loop (VDLL) (Pany et al. 2005) is used in conjunction with conventional carrier frequency tracking. The VDLL tracks code for all signals and maintains the navigation solution. Pseudorange rate measurements from the frequency tracking loops aid both the code tracking and the navigation solution computation. The key difference between the VDLL and conventional code tracking is that the code numerically controlled oscillator (NCO) commands, used to align the receiver-generated reference codes with the incoming signals, are generated using information from all of the signals tracked. Where more than four signals are tracked, this increases robustness against signal attenuation and interference. It can also prevent the receiver locking onto long-path-delay NLOS signals, which are responsible for the very large positioning errors $(>100 \mathrm{~m})$ that sometimes occur in dense urban areas. The VDLL implemented here also uses innovation-based adaptive estimation (Mehra 1972; Mohammed and Schwarz 1999) to determine the EKF measurement noise, instead of using carrier-power-to-noise density ratio $\left(C / N_{0}\right)$ or signal amplitude estimates. $C / N_{0}$ and signal amplitude are difficult to determine in weak signal and high interference environments 
(Groves 2005).

The proposed scheme is evaluated against an equivalent conventional architecture comprising separate code tracking loops and an EKF-based navigation solution. In some of the tests, a strobe correlator (Garin et al. 1996; Veitsel et al. 1998), a receiver-based multipath mitigation technique, is also used with both the conventional and vector tracking architectures, enabling a four-way performance comparison to be made. As the aim here is to assess multipath and NLOS mitigation, the atmospheric propagation errors are minimized using the Saastamoinen tropospheric model (Saastamoinen 1972), the UNB3 model (LaMance et al. 1996), and the International GNSS Service (IGS) exact ionospheric model (Hernández-Pajares et al. 2009).

This paper firstly describes the vector tracking algorithm, which has been implanted on a Matlab-based GPS software-defined receiver. In the section of simulation results, the vector tracking algorithm us tested under a long-path-delay NLOS reception situation. Then, a Monte-Carlo simulation is used to test its sensitivity with and without the strobe correlator. In the section of experiment, this paper presents the results of a road vehicle experiment conducted in a high-multipath and NLOS environment in central Tokyo. The GNSS signals were sampled and recorded to enable vector tracking to be compared with conventional tracking, with and without the strobe correlator, using the same data set. The capability of vector tracking to mitigate multipath interference and NLOS reception is evaluated.

\section{Vector tracking algorithm}

This section describes the vector tracking algorithm used for this study. The overall architecture is described first, then the EKF, including the adaptive tuning algorithm. This is followed by a brief summary of the conventional tracking algorithm used for comparison. 
Vector tracking architecture

The architecture of the VDLL implemented is displayed in Figure 1. Note that a conventional tracking architecture is used for initialization, providing code phase and Doppler frequency estimates for signals from at least four satellites together with satellite ephemeris data and a position, velocity, and time (PVT) solution. 


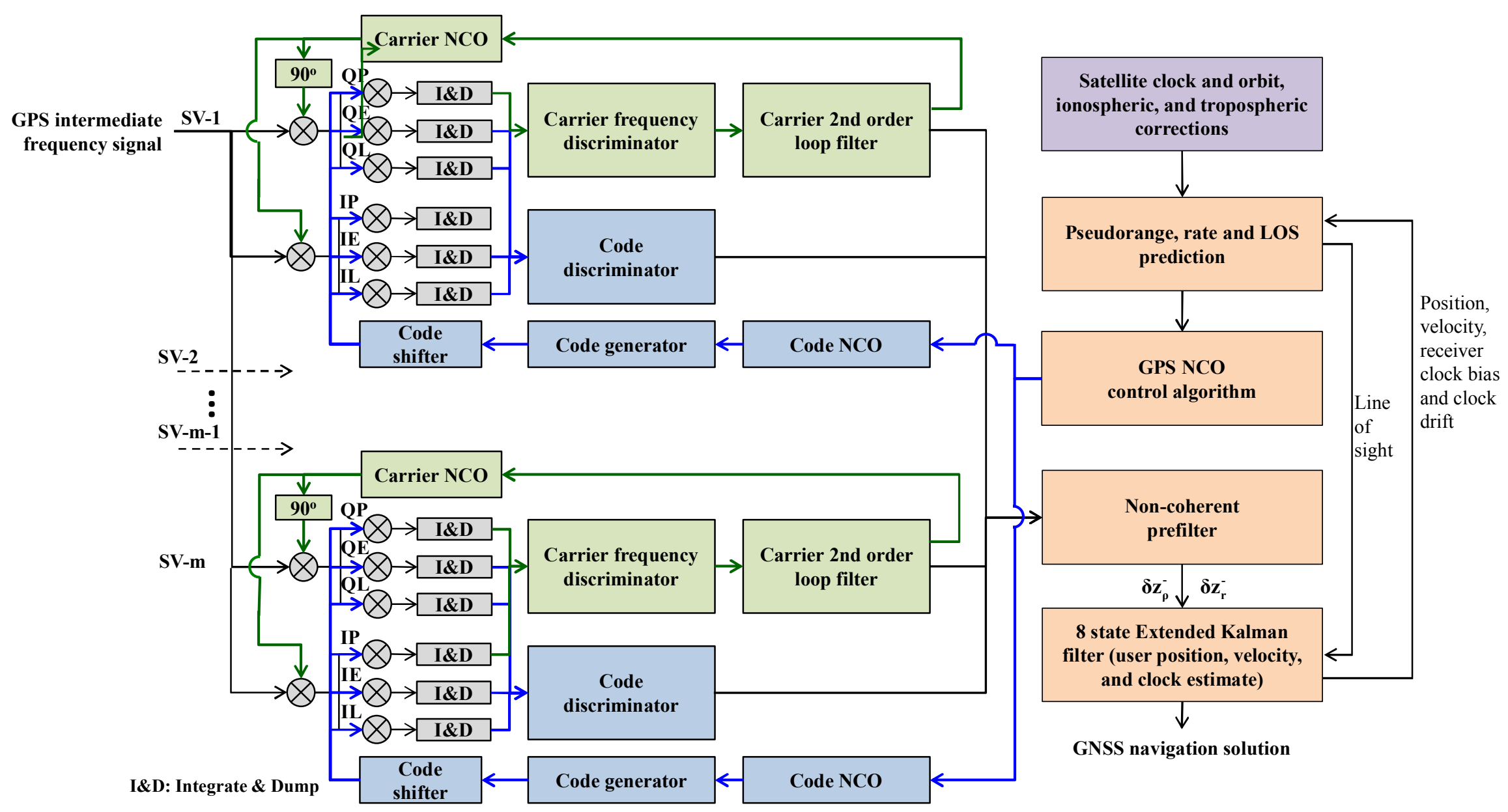

Figure 1: Detailed architecture of the developed VDLL 
In Figure 1, the blue and green parts components comprise code and carrier tracking, respectively. Both code and carrier tracking includes a discriminator, loop filter, and NCO. The orange components comprise the navigation algorithms including non-coherent prefilters, an EKF, prediction of the pseudoranges and GPS code NCO command generation. As shown, within each channel, the incoming signal is correlated with six locally-generated replica signals: in-phase (I) and quadraphase $(\mathrm{Q})$ early $(\mathrm{E})$, prompt $(\mathrm{P})$, and late $(\mathrm{L})$. The correlator outputs are then input to the code and carrier discriminator functions. For carrier tracking, arctangent frequency discriminators are used with a separate $2^{\text {nd }}$ order frequency lock loop (FLL) with a $5 \mathrm{~Hz}$ noise bandwidth for each signal tracked, which outputs the estimated Doppler shift to the navigation algorithms. For code, early-minus-late envelope discriminators with a one chip early-late spacing are used; these output directly to the navigation algorithms. Further details may be found in standard texts (Ward et al. 2006b; Misra and Enge 2011; Groves 2013).

To apply correlator-based multipath mitigation techniques, the standard code discriminator is replaced and additional or alternative correlators employed, where required. Here, a strobe correlator option has been implemented (Garin et al. 1996; Veitsel et al. 1998). Where this is selected, the early and late correlators are replaced by strobe correlators in which the incoming signal is correlated with a linear combination of reference signals subject to different delays and the code discriminator is replaced with

$$
d_{\text {strobe }}=\frac{I D \cdot I P+Q D \cdot Q P}{I P^{2}+Q P^{2}}
$$

where $I D$ and $Q D$ are, respectively, the in-phase and quadrature components of the strobe correlators and $I P$ and $Q P$ are, respectively, the in-phase and quadrature components of prompt code correlators.

An EKF is used to correct the position and velocity solution using the code discriminator outputs and Doppler shift estimates. However, it is neither necessary nor practical to iterate the EKF at the same rate as the accumulated correlator outputs are updated. Therefore, pre-filters are used as an intermediary, averaging the code discriminator outputs over the 20 ms EKF update interval. Averaging of the Doppler measurements is not required in the pre-filter because the FLLs already do this. Note that if the integration time of FLL is shorter than $20 \mathrm{~ms}$, the averaging is required for the FLL output as well. The measurements input to 
the EKF are delta pseudorange, $\Delta \rho^{j}$ (in meters), and pseudorange rate, $\dot{\rho}^{j}$ (in meters per second). The delta pseudorange is the difference between the true and predicted pseudorange. They are calculated in the pre-filter using:

$$
\begin{aligned}
& \Delta \rho^{j}=d^{j} \times \frac{c}{f_{0}} \\
& \dot{\rho}^{j}=f_{\text {Doppler }}^{j} \times \frac{c}{f_{L 1}}
\end{aligned}
$$

where $d$ is the code discriminator output in chips, $f_{\text {Doppler }}$ is the Doppler frequency in $\mathrm{Hz}, c$ is the speed of light, $f_{0}$ is the code chipping rate (1.023 MHz for GPS C/A code), and $f_{L I}$ is the L1 band carrier frequency (1575.42 MHz).

The EKF estimates corrections to the position, velocity and receiver clock solution and is described in more detail in the next sub-section. The corrected receiver velocity and clock drift solution are used to update the position and clock offset every $20 \mathrm{~ms}$, from which each of the pseudo-ranges are predicted using

$$
\hat{\rho}_{R, k}^{j}=\sqrt{\left[\hat{r}_{s}^{j}-\hat{r}_{a}^{-}\right]^{T}\left[\hat{r}_{s}^{j}-\hat{r}_{a}^{-}\right]}+\delta \hat{\rho}_{s v, c}^{j}+\delta \hat{\rho}_{T}^{j}+\delta \hat{\rho}_{I}^{j}-\hat{b}_{k}
$$

where $\hat{r}_{s}^{j}$ is the Cartesian earth-centered inertial (ECI) frame position of the satellite denoted by index $j$, determined from the broadcast ephemeris, $\hat{r}_{a}^{-}$is the predicted ECI-frame receiver position, $\delta \hat{\rho}_{s v, c}^{j}$ is the satellite clock correction, $\delta \hat{\rho}_{T}^{j}$ and $\delta \hat{\rho}_{I}^{j}$ are, respectively, the tropospheric and ionospheric error estimates, and $\hat{b}_{k}$ is the estimated receiver clock bias. The predicted pseudo-ranges are then used to generate the code NCO commands using

$$
\hat{f}_{c o, N C O j, k+1}^{j}=f_{0}\left[1-\frac{\left(\hat{\rho}_{R, k+1}^{j}-\hat{\rho}_{R, k}^{j}\right)}{c \tau_{a}}\right]
$$

where $\tau_{a}=20 \mathrm{~ms}$ is the interval between epochs $k$ and $k+1$. The NCO commands then control the receiver's replica code generators for all of the signals, closing the vector delay lock loop. 
Extended Kalman filter

The extended Kalman filter is the standard algorithm used to compute a multi-epoch GNSS navigation solution. A full description may be found in standard navigation texts (Brown and Hwang 1996; Jwo et al. 2009; Groves 2013). Here, only the details specific to the vector tracking implementation are presented. The EKF estimates the errors in the position, velocity, and clock solution used to control the receiver replica code generator. The state vector, $\mathbf{x}$, thus comprises the following states:

$$
\boldsymbol{x}^{T}=\left[\begin{array}{llllllll}
\Delta p_{x k} & \Delta p_{y k} & \Delta p_{z k} & \Delta v_{x k} & \Delta v_{y k} & \Delta v_{z k} & \Delta b_{k} & \Delta d_{c l k, k}
\end{array}\right]
$$

where $\Delta \mathbf{p}_{k}, \Delta \mathbf{v}_{k}, \Delta b$ and $\Delta d_{c l k}$ are the position, velocity, receiver clock bias and drift, respectively. The position and velocity errors are resolved in and referenced to an earth-centered earth-fitted (ECEF) frame and the clock bias and drift are expressed as a range and range rate, respectively. All states are initialized at zero. The transition matrix, used for the time propagation of the states, is

$$
\boldsymbol{\Phi}=\left[\begin{array}{ccc}
\mathbf{I}_{3 \times 3} & \tau_{s} \mathbf{I}_{3 \times 3} & 0_{3 \times 2} \\
0_{3 \times 3} & \mathbf{I}_{3 \times 3} & 0_{3 \times 2} \\
0_{2 \times 3} & 0_{2 \times 3} & \boldsymbol{\Phi}_{\mathbf{c l k}}
\end{array}\right]_{8 \times 8}, \boldsymbol{\Phi}_{\mathbf{c l k}}=\left[\begin{array}{cc}
1 & \tau_{s} \\
0 & 1
\end{array}\right]
$$

where $\tau_{s}$ is the EKF update interval $(20 \mathrm{~ms})$.

The system (or process) noise covariance matrix may be divided into user dynamic noise and receiver clock noise as follows:

$$
\mathbf{Q}=\left[\begin{array}{cc}
\mathbf{B}_{\text {dynamic }} & 0_{6 \times 2} \\
0_{2 \times 6} & \mathbf{B}_{\text {clock }}
\end{array}\right]
$$

where

$$
\mathbf{B}_{\text {dynamic }}=\left[\begin{array}{cc}
\tau_{s}^{3} / 3 \cdot \mathbf{I}_{3 \times 3} & \tau_{s}^{2} / 2 \cdot \mathbf{I}_{3 \times 3} \\
\tau_{s}^{2} / 2 \cdot \mathbf{I}_{3 \times 3} & \tau_{s} \cdot \mathbf{I}_{\mathbf{3} \times \mathbf{3}}
\end{array}\right] \cdot S_{v}
$$




$$
\mathbf{B}_{\text {clock }}=\left[\begin{array}{cc}
S_{c \phi} \tau_{s}+\frac{1}{3} S_{c f} \tau_{s}^{3} & \frac{1}{2} S_{c f} \tau_{s}^{3} \\
\frac{1}{2} S_{c f} \tau^{2} & S_{c f} \tau_{s}
\end{array}\right],\left\{\begin{array}{c}
S_{c \phi}=c^{2} \cdot \frac{h_{0}}{2} \\
S_{c f}=c^{2} \cdot 2 \pi^{2} \cdot h_{-2}
\end{array}\right.
$$

where $S_{v}$ is the user velocity noise power spectral density (PSD), $S_{c \varphi}$ is the oscillator phase noise PSD, and $S_{c f}$ is the oscillator frequency noise PSD. Here, $S_{v}$ is set to $1 \mathrm{~m}^{2} / \mathrm{s}^{3}$. The front-end is equipped with a temperature compensate crystal oscillator (TCXO), so suitable clock model coefficients are (Brown and Hwang 1996):

$$
\begin{aligned}
& h_{0}=2 \times 10^{-19} \\
& h_{-2}=2 \times 10^{-20}
\end{aligned}
$$

The measurement innovations input to the EKF comprise the delta pseudo-ranges, obtained directly from the pre-filter, and the delta pseudorange rates, comprising the differences between the measured pseudorange rates from the pre-filter and the predicted values computed from the user and satellite velocities and clock drifts. Thus, the measurement innovation vector is

$$
\boldsymbol{\delta} \mathbf{z}_{\mathbf{k}}^{-}=\left[\begin{array}{llllllll}
\Delta \rho_{k}^{1} & \Delta \rho_{k}^{2} & \cdots & \Delta \rho_{k}^{m} & \Delta \dot{\rho}_{k}^{1} & \Delta \dot{\rho}_{k}^{2} & \cdots & \Delta \dot{\rho}_{k}^{m}
\end{array}\right]^{T}
$$

where $\Delta \rho_{k}^{j}$ and $\Delta \dot{\rho}_{k}^{j}$ are, respectively, the delta pseudorange and delta pseudorange rate for satellite $j$ at epoch $k$. The measurement matrix, which comprises the partial derivatives of the measurements with respect to the states is

$$
\mathbf{H}=\left[\begin{array}{cccc}
\mathbf{U} & 0_{m \times 3} & 1_{m \times 1} & 0_{m \times 1} \\
0_{m \times 3} & \mathbf{U} & 0_{m \times 1} & 1_{m \times 1}
\end{array}\right], \mathbf{U}=\left[\begin{array}{ccc}
-u_{x}^{1} & -u_{y}^{1} & -u_{z}^{1} \\
-u_{x}^{2} & -u_{y}^{2} & -u_{z}^{2} \\
\vdots & \vdots & \vdots \\
-u_{x}^{m} & -u_{y}^{m} & -u_{z}^{m}
\end{array}\right]
$$

where $m$ is the number of satellites, $\mathbf{u}$ is the line-of-sight (LOS) unit vector from the receiver to the satellite, its subscript denotes the $x, y$, and $z$ components, and its superscript denotes the satellite. 
The measurement noise covariance matrix, $\mathbf{R}$, is determined adaptively, with the off-diagonal terms assumed to be zero because of the weak correlation between the different PRN codes and the orthogonality of the code and carrier discriminator noise. A variation of the innovation-based adaptive estimation (IAE) technique (Mehra 1972; Mohammed and Schwarz 1999) is used. The variance of each component of the measurement innovation is calculated over the last 500 epochs. Each value is then multiplied by a scaling factor to form the corresponding diagonal element of $\mathbf{R}$. The scaling factor accounts for the time correlation of the measurement noise. This scaling factor only used in carrier measurement because the VDLL is selected in this work. For the carrier measurements, a scaling factor of 10 is used because the EKF update rate is 10 times the bandwidth of the carrier frequency tracking loops. To prevent filter instability, minimum and maximum values of each component of $\mathbf{R}$ are imposed. The maximum and minimum values are 5,000 and $100 \mathrm{~m}^{2}$, respectively, for code measurement and 50 and $0.01 \mathrm{~m}^{2} / \mathrm{s}^{2}$, respectively, for carrier measurement. These values were determined empirically using data from urban environments. This adaptive estimation technique automatically downweights measurements that are noisy, not only because of a low $C / N_{0}$, but also due to the effects of rapidly fluctuating multipath interference, as shown in the section of experiment result.

Equivalent conventional tracking architecture

The aim of the investigation is the performance of vector tracking against multipath interference and NLOS reception. Therefore, the design of the conventional receiver architecture used for comparison must be as close as possible to the vector tracking architecture. Thus, the correlator spacing, coherent integration time, type of discriminator and carrier tracking loop are the same for both the vector and conventional architectures. The conventional architecture also uses an EKF-based navigation processor. This has the same states, system model, measurement model, and noise models as the vector-tracking EKF, including adaptive measurement noise covariance. The ionospheric and tropospheric models are also the same. However, there are two major differences between the conventional and vector tracking architectures. The first difference is that the conventional receiver uses a separate $2^{\text {nd }}$ order loop filter to track the code of each signal, calculating the NCO command. Thus, it tracks all signals independently. The second difference is in the calculation of code measurement, specifically the formation of the delta pseudorange measurement innovations. These are formed by subtracting the pseudo-ranges predicted from the user and satellite PVT 
solutions from the pseudo-ranges estimated from the code tracking loops.

\section{Simulation test of vector tracking in NLOS reception}

Simulation tests were conducted to assess the response of vector and conventional tracking to long-path-delay NLOS reception and to compare the sensitivity of the different techniques. Each is described in turn.

Simulation of exaggerated NLOS reception

To simulate relatively strong NLOS reception with a long path delay in the intermediate frequency (IF) signal samples, an algorithm called direct signal cancellation was implemented (Hsu et al. 2007). This takes the IF signal recorded by a GNSS front end, subtracts a replica of one of the signals from this data and adds a delayed version of the same replica signal to simulate NLOS reception. The amplitude, code phase, carrier phase and Doppler shift of the replica signal are determined by tracking the original signal from the unmodified IF data. Figure 2 illustrates the process.

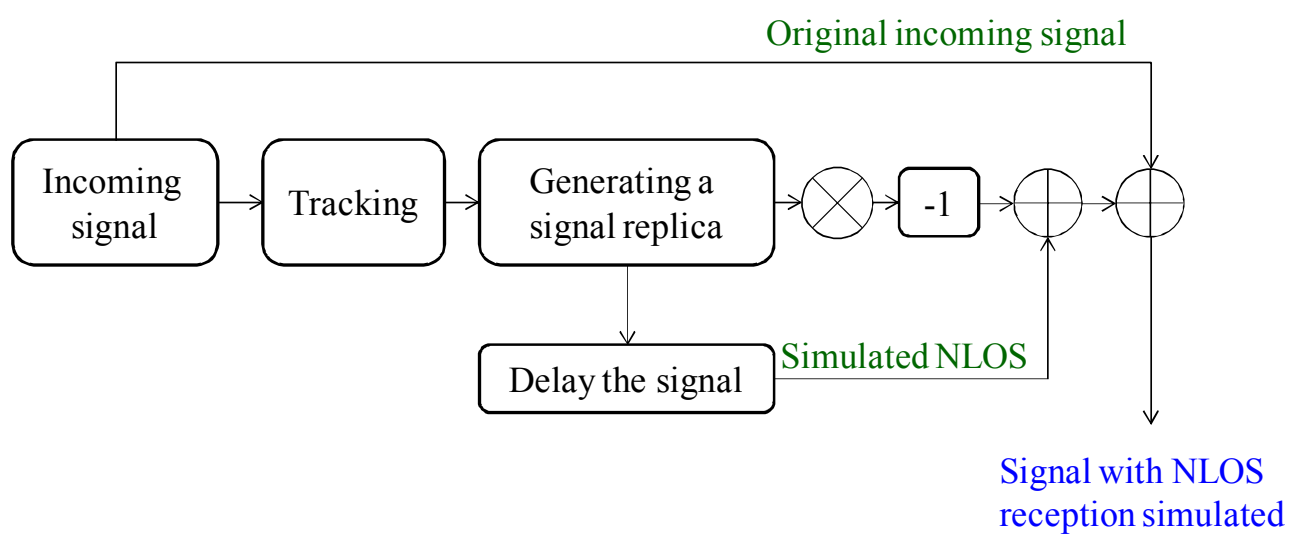

Figure 2: Simulation of NLOS reception using on the direct signal cancellation method.

GPS signals for use in the NLOS simulations were recorded statically on the roof of the fourth research building at the Etchujima campus of Tokyo University of Marine Science and Technology (TUMSAT). Signals from 9 satellites were tracked. Between 6 and 10s from the start of the run, the signal from GPS PRN27 was eliminated from the recorded IF data and a simulated reflected signal from the same satellite added in its place. For the rest of the run, 
the original PRN27 signal was retained. The simulated reflected signal was $8 \mathrm{~dB}$ weaker than the direct line of sight (LOS) signal it replaced and was delayed by 0.8 chips relative to the direct path. Figure 3 shows the correlator and code discriminator outputs for PRN27 using both conventional and vector tracking.
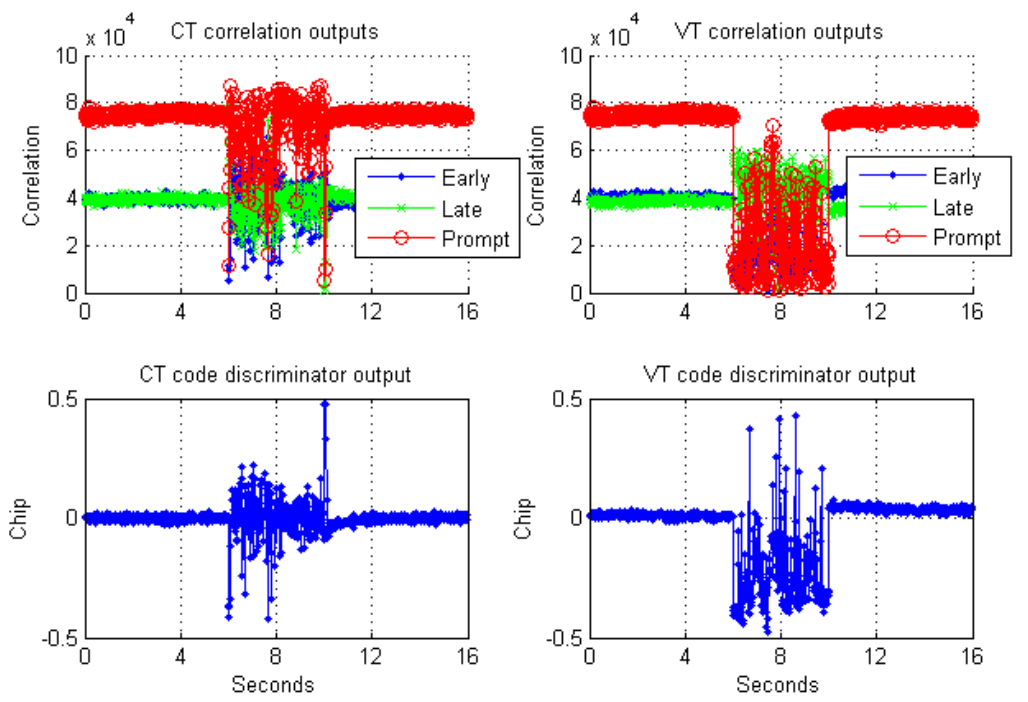

Figure 3: Correlator and code discriminator outputs for PRN27 using conventional tracking (CT) and vector tracking (VT) with NLOS reception simulated from 6 to 10s.

Examining the conventional-tracking code discriminator output, anomalies occur mainly at the beginning and end of the period of NLOS reception. The correlation value in the prompt channel is also generally the highest among the three during the period of NLOS reception. Thus, the conventional-tracking loop is locking onto the NLOS signal. Consequently, it is difficult to detect NLOS reception from the correlator outputs in a conventional-tracking architecture.

The vector-tracking code discriminator output has a large negative value during most of the period of NLOS reception. Furthermore, the highest correlation value is found in the late channel. Both of these phenomena provide an indication of potential NLOS reception. This happens because, in vector tracking, the receiver-generated code in each correlation channel is determined using the user and satellite PVT solutions. Consequently, when the user PVT solution is overdetermined, the prompt reference code is not aligned with an NLOS signal. Instead, it is aligned with the predicted direct signal, which is blocked in the case of NLOS reception. However, NLOS reception can still contaminate the navigation solution because inputting an NLOS code discriminator measurement to the vector-tracking EKF will 
pull the position solution away from the truth. It is therefore desirable to detect NLOS signals and exclude their measurements from the vector-tracking EKF, noting that correlation of NLOS signals can continue using NCO commands generated using the navigation solution. In order to detect NLOS reception, the code discriminator output is used to test the quality of the signal. If the code discriminator output exceeds a pre-determined value, threshold_chip, an abnormal output is assumed and a counter incremented by one. The counter is reset to zero after $N$ epochs without any increments, defining a detection window. If the counter value exceeds the pre-determined threshold, $N \times$ threshold_percent, NLOS reception is suspected. Once a signal is suspected to be NLOS, its measurements are excluded from the EKF. For the results presented here, $N$ was set to 10020 -ms epochs, giving a 4 s detection window, threshold_chip was set to 0.2 chips and threshold_percent set to 0.25 (i.e., 25\%). Both two thresholds were determined by trial and error.

Figure 4 shows the 3D positioning root mean square error (RMSE) of conventional tracking, vector tracking, and vector tracking with the NLOS detection technique. Without NLOS detection, the positioning accuracy of both conventional and vector tracking is significantly degraded as a result of the $\sim 240 \mathrm{~m}$ ranging error of the simulated NLOS signal. The positioning error of vector tracking with NLOS detection increases slightly. The PDOP values before and after the NLOS signal is detected are 2.46 and 4.65 , respectively. Thus, the increase in position error can be attributed to the degradation in satellite geometry that results from eliminating one of the signals. Figure 5 shows the velocity error in each case. The conventional solution develops a significant time-correlated bias when the NLOS signal is simulated, while the basic vector-tracking solution is very noisy. With NLOS detection and exclusion, the velocity solution is smoother and more accurate. Thus, vector tracking with NLOS detection clearly gives the best navigation solution of the three. Note that this technique does not correct NLOS reception errors; it only excludes the affected signals from the position solution. It also requires at least four uncontaminated GPS signals to form a good position solution. Thus, it will not work under all conditions. The technique's impact will also be less for weak NLOS signals as weak signals already receive lower weighting in the EKF. 


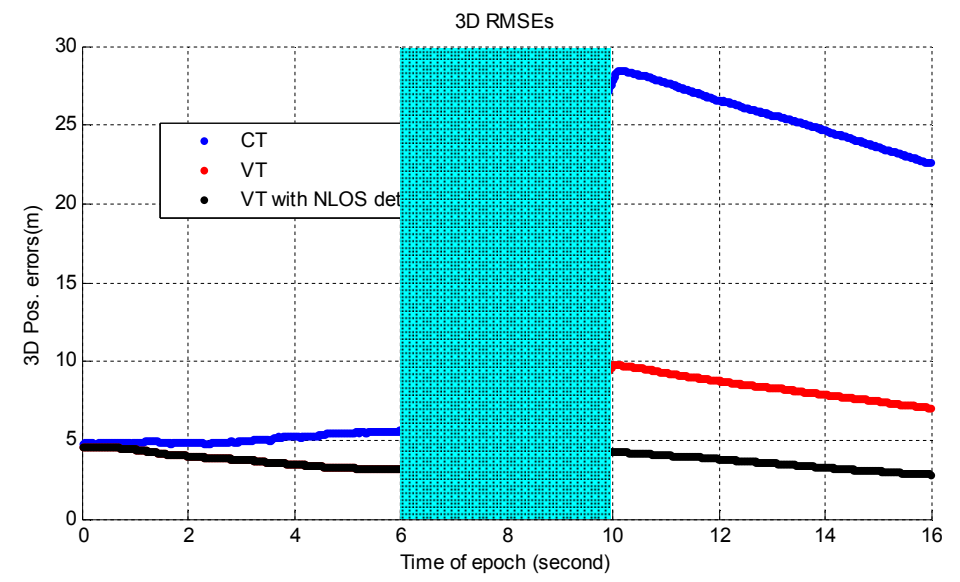

Figure 4: The 3D positioning RMSE of CT, VT, and VT with NLOS detection technique. The pink shadow represents the duration of simulated NLOS reception.
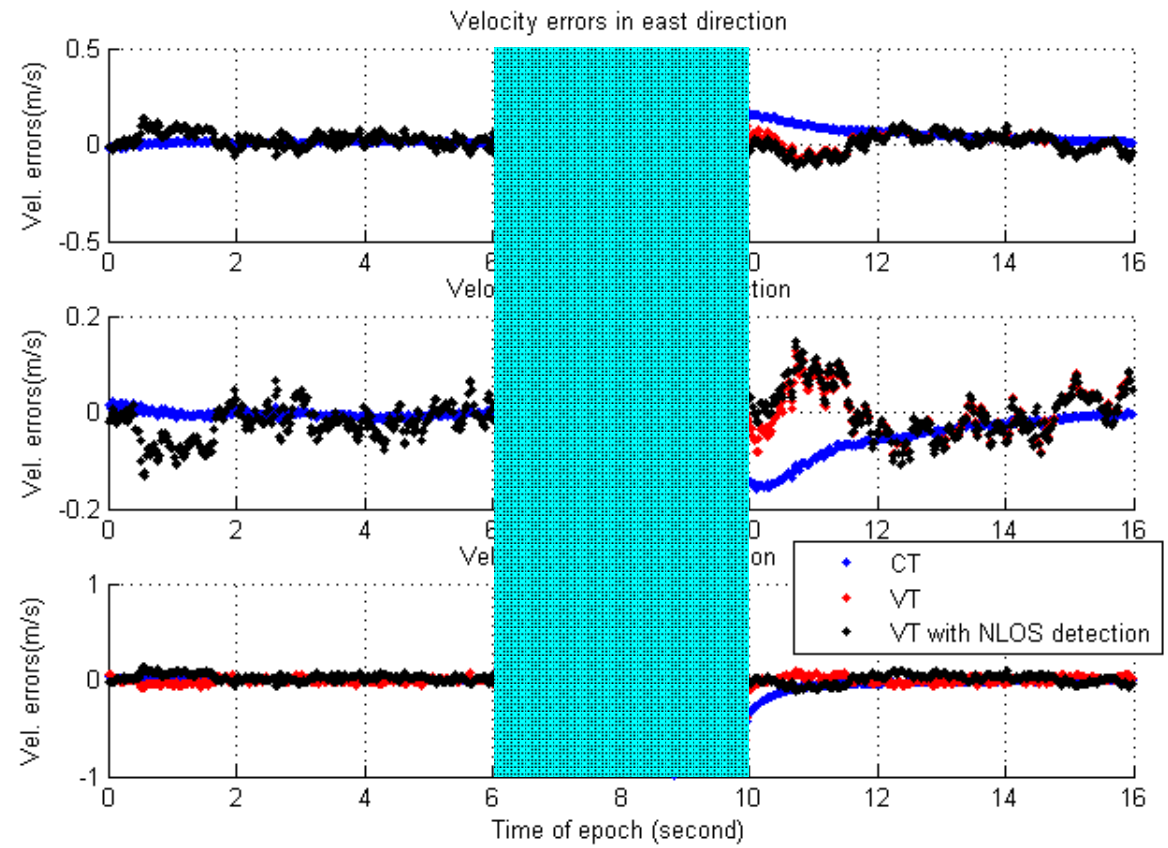

Figure 5: The 3D velocity error of CT, VT, and VT with NLOS detection technique. The pink shadow represents the duration of simulated NLOS reception.

Monte Carlo Sensitivity Test

Receiver-based multipath mitigation techniques, such as the strobe correlator, effectively enhance the resolution of the code discriminator for low-chipping-rate signals by boosting the higher frequency components of the signal modulation. However, as the higher frequency components are weaker, this reduces the sensitivity of the receiver (Pratt 2004). These higher 
resolution code discriminators also have narrower pull-in regions, so code tracking lock is lost as a result of smaller tracking errors (Garin et al. 1996).

To assess the sensitivity loss from using the strobe correlator, a series of Monte Carlo simulations were conducted to determine the code tracking thresholds of both conventional and vector tracking with and without the strobe correlator. Details are presented in (Hsu 2013). It was found that the strobe correlator increases the conventional code tracking threshold by $\sim 2.3$ $\mathrm{dB}$ (i.e. the receiver sensitivity is reduced by $2.3 \mathrm{~dB}$ ), whereas the threshold for vector code tracking is only increased by $\sim 1.4 \mathrm{~dB}$ when the strobe correlator is used. This is thought to be because vector tracking can recover signals that briefly leave the discriminator pull-in range using the navigation solution derived from the other signals.

\section{Experiments}

Preliminary experiments were conducted at the Tzu-Chang campus of National Cheng Kung University in Taiwan. GNSS signals were recorded on a pedestrian route in an open area surrounded by buildings. Both multipath interference and NLOS reception were present along many parts of the route. Details are described in (Hsu et al. 2013; Hsu 2013). Without the strobe correlator or the NLOS receiver, the horizontal RMSE across the whole circuit was $1.51 \mathrm{~m}$ with vector tracking, compared to $2.60 \mathrm{~m}$ with conventional tracking, a clear improvement. Here, the strobe correlator degraded the RMSE across the whole run to $1.60 \mathrm{~m}$ with vector tracking. However, it improved the positioning performance at the locations most severely affected by multipath interference.

Experiments were conducted in an urban canyon in the Toyusu area of Tokyo, shown in Figure 6. The equipment used is shown in Figure 7. For the first experiment, GNSS signals captured by a NovAtel 702 antenna were sampled and recorded using a Fraunhofer front-end, which has a precorrelation bandwidth of $13 \mathrm{MHz}$, an IF of $12.82 \mathrm{MHz}$, and a sampling frequency of $40.96 \mathrm{MHz}$. The reference was provided by a NovAtel OEM6 receiver, which shared the antenna with the Fraunhofer front-end via a GPS Networking antenna splitter. The antenna was attached on the top of an automobile as shown in the left of Figure 7. The signal data was collected statically for about 5 minutes at the position marked by a pin in Figure 6. 


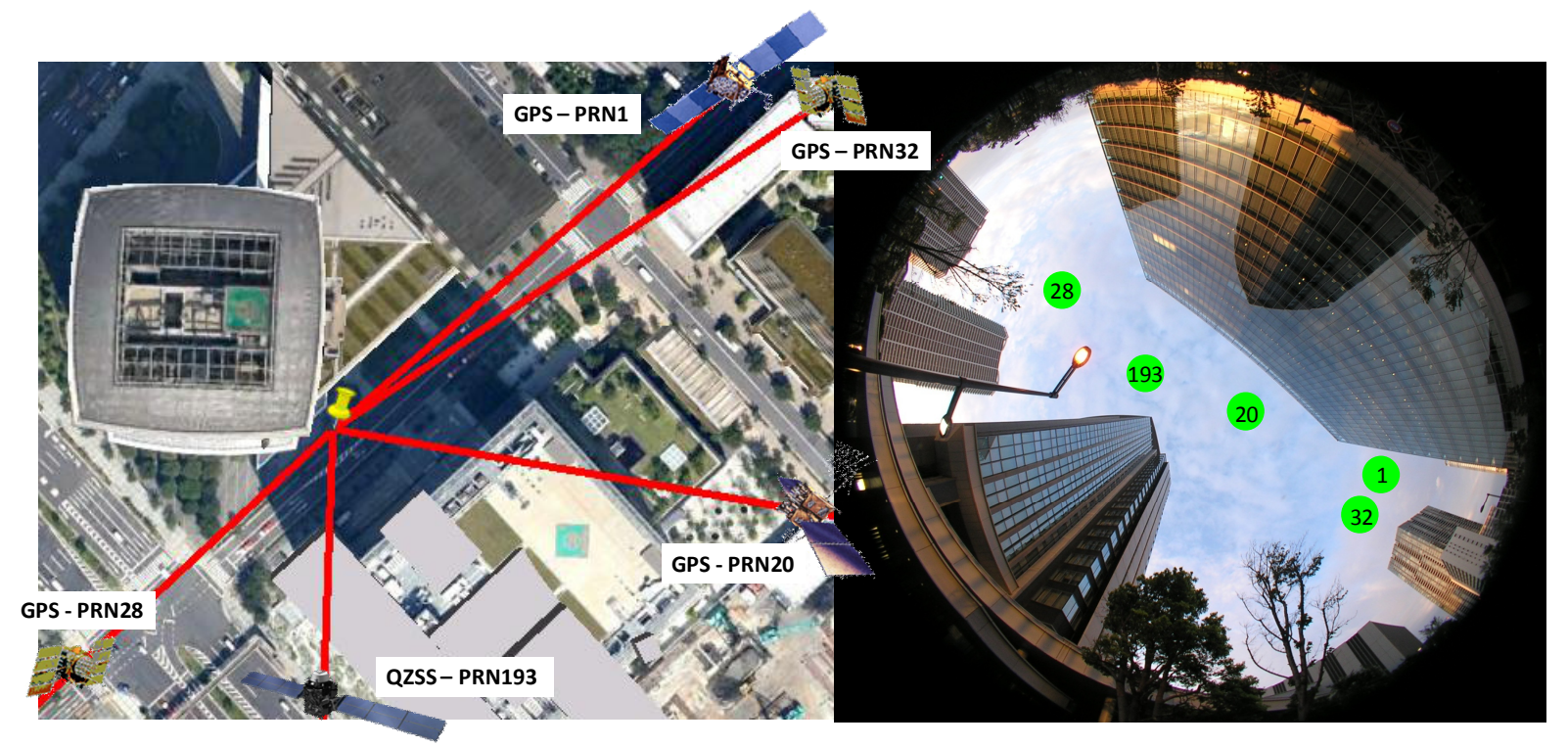

Figure 6: Bird-view and fisheye-view of the selected urban canyon in Toyosu area.
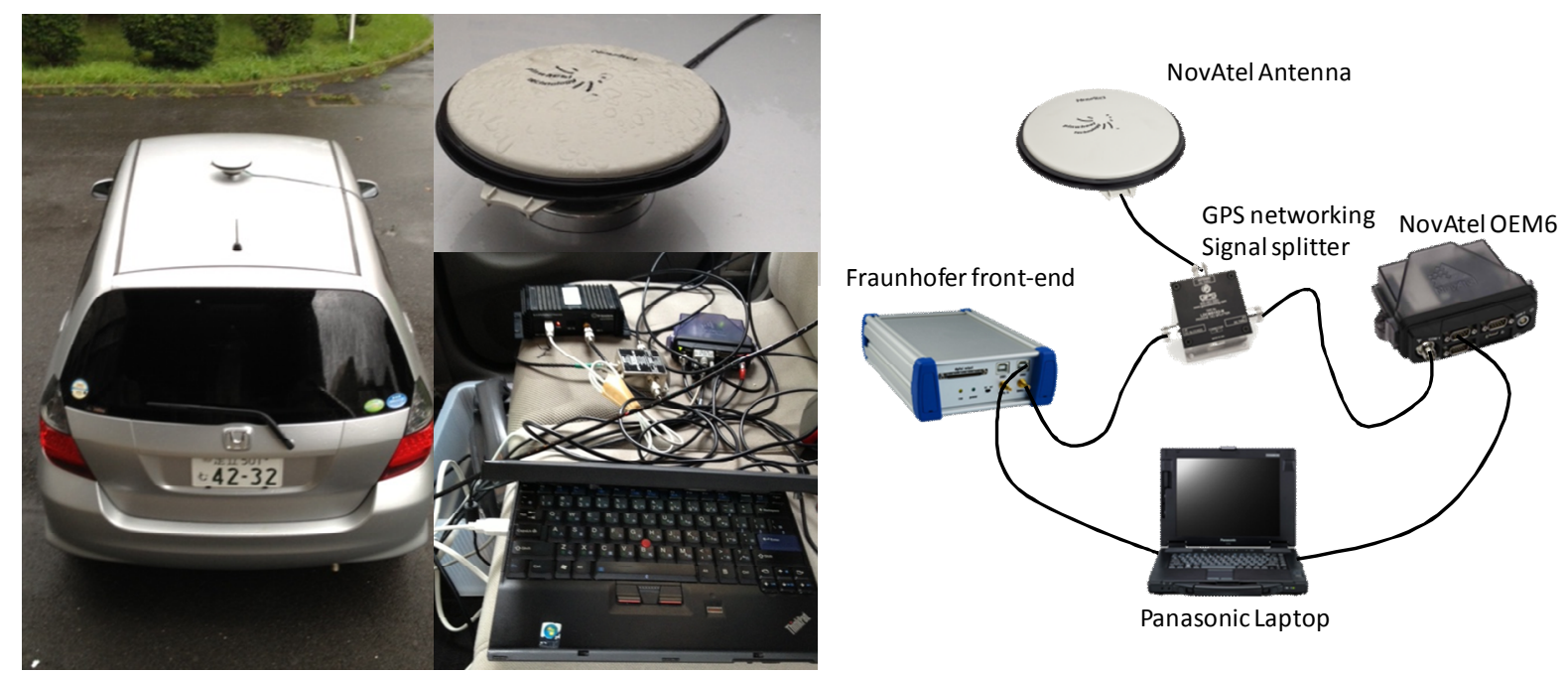

Figure 7: Equipment used in the Tokyo city experiments.

In the left part of Figure 6, the red lines depict the LOS vectors between the satellites and the user antenna. In this experiment, only 5 satellites could be continuously tracked due to the very narrow open sky as shown in the right of Figure 6 . Of these, four are GPS satellites and the fifth a Quasi-Zenith Satellite System (QZSS) satellite. The surrounded buildings are covered by very flat glass, which is a strong reflector to GNSS signals; sometimes attenuating them by as little as $2-3 \mathrm{~dB}$ (Groves 2013). The $C / N_{0}$ measurements from the software receiver are shown in Figure 8. Significant fluctuation can be seen in all of the $C / N_{0}$ measurements, except for the QZSS satellite, which indicates that these signals are affected by strong multipath interference. PRN1 was the most affected satellite. An 
example of the PRN1 code correlation function is also shown in Figure 8. The distortion of the correlation function away from a symmetrical triangle can be clearly seen in the middle of Figure 8 . The regular correction shape is also shown in the bottom of Figure 8.
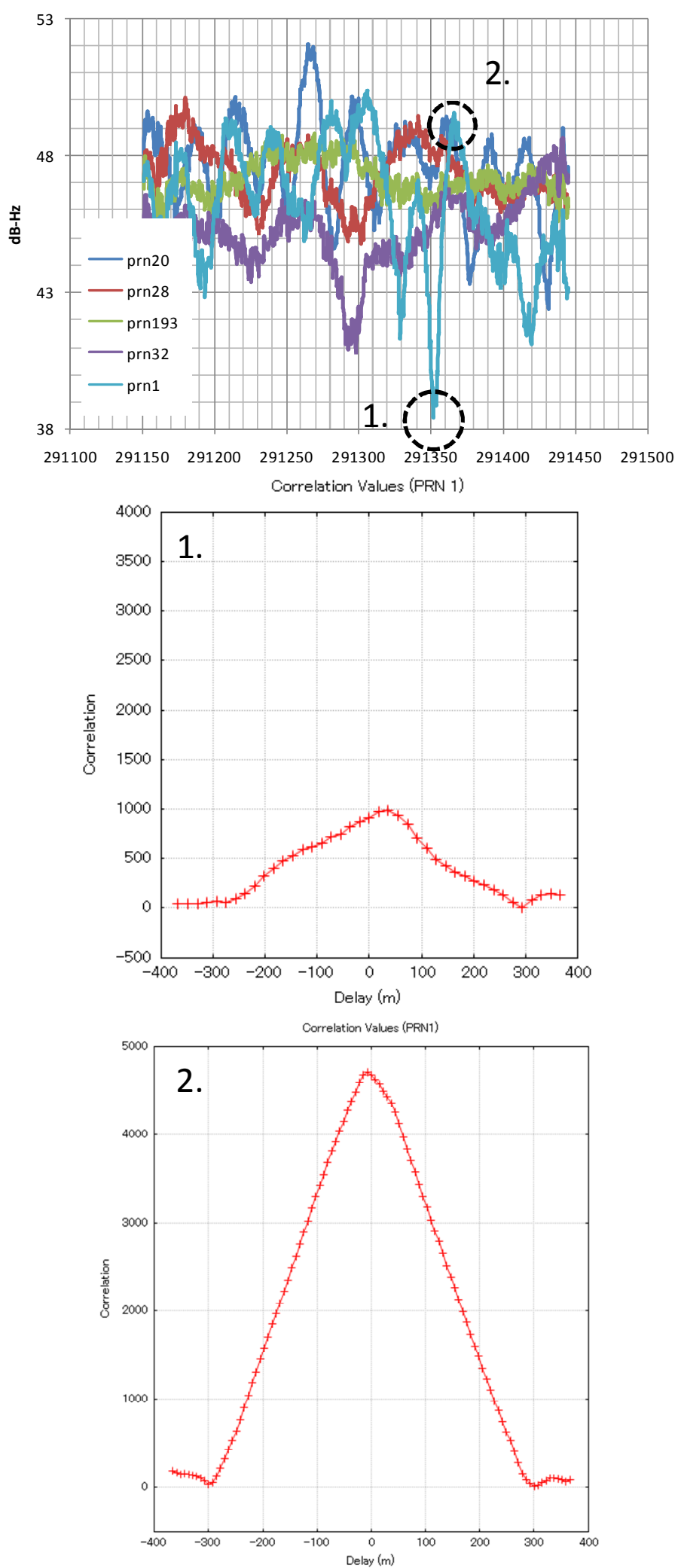
Figure 8: The calculated $C / N_{0}$ of the experiment of urban canyon is shown in upper plot and the correlation shape of the normal case and multipath effect are shown in lower plot.

The vector-tracking results are compared not only with the equivalent conventional-tracking receiver, but also an open-source real time GNSS software receiver, called GNSS-SDRLIB (www.taroz.net/gnsssdrlib_e.html). This open-source receiver implements a single-epoch positioning algorithm and supports carrier smoothing, differential GPS (DGPS) and the strobe correlator. The carrier-smoothing time constant for the open-source receiver was 100 seconds, while the strobe correlator spacing in both receivers was \pm 0.3 chips and \pm 0.15 chips. The baseline for differential positioning using the open-source software receiver was less than one kilometer.

Figure 9 shows the positioning results from the Toyosu experiment obtained with different receiver configurations. The positioning errors are mainly along a north-west south-east axis because of the satellite geometry (see Figure 6). It is interesting to note the positioning results using vector tracking (red star) are much better than with the equivalent conventional tracking (blue cross), while the strobe correlator only slightly improves the vector-tracking results.

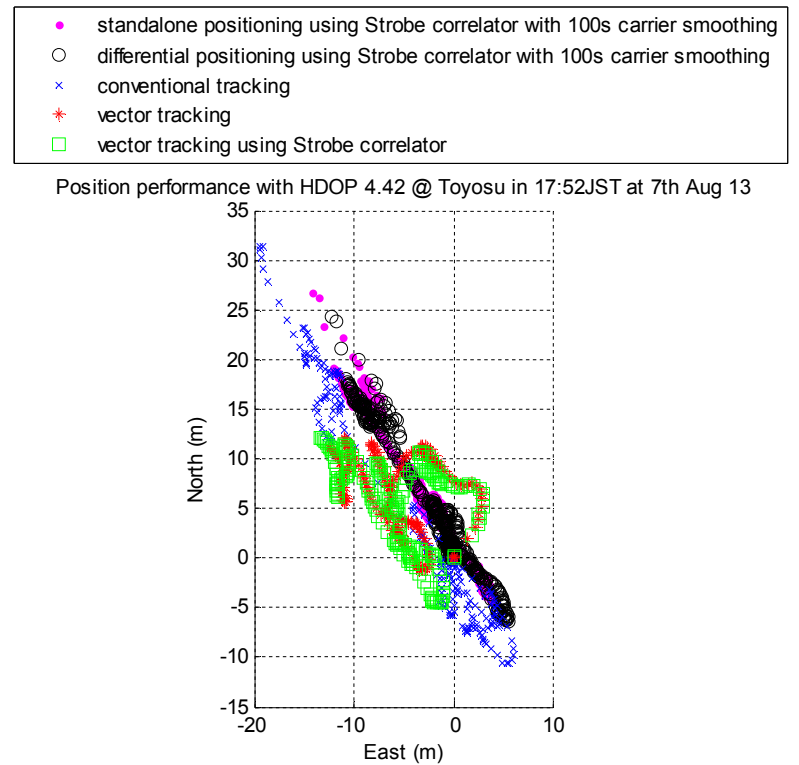

Figure 9: Position errors with the Fraunhofer front-end and different receiver configurations in Toyosu, Tokyo. The horizontal dilution of precision (HDOP) is 4.42 in all positioning cases which indicates a poor satellite geometry. 
Table 1 lists the positioning performance of different approaches. Differential positioning using the strobe correlator and gives the smallest mean position error, showing that the atmospheric and signal-in-space errors are significant. However, the smallest error standard deviation is obtained using vector tracking. Vector tracking also outperforms the equivalent conventional tracking configuration by about $2 \mathrm{~m}$ and $4.3 \mathrm{~m}$ in terms of the mean and standard deviation, respectively. Vector tracking thus stabilizes the position solution in the presence of multipath interference. This effect can be seen in Figure 10, which shows the code discriminator output from both conventional and vector tracking. As vector tracking is capable of estimating a more stable code frequency, the effects of multipath interference can be observed on its code discriminator outputs. Periodic oscillation can be seen for PRN1, PRN28 and PRN30, while a peak error can be seen in the PRN1 data. This could be due to a passing vehicle temporarily blocking the direct LOS signal, resulting in a short period of NLOS reception.

Table 1: 3D position error statistics with the Fraunhofer front-end and different receiver configurations in Toyosu, Tokyo

\begin{tabular}{l|c|c}
\hline \multicolumn{1}{c|}{ Approaches } & $\begin{array}{c}\text { Mean of 3D position } \\
\text { error (meters) }\end{array}$ & $\begin{array}{c}\text { Standard Deviation of } \\
\text { 3D position error } \\
\text { (meters) }\end{array}$ \\
\hline $\begin{array}{l}\text { 1. Single-epoch positioning } \\
\text { 2. Single-epoch positioning with carrier }\end{array}$ & 30.20 & 20.24 \\
smoothing & 12.34 & 7.10 \\
3. Single-epoch positioning using a strobe & 19.47 & 6.52 \\
correlator & & 6.18 \\
4. Single-epoch positioning using a strobe & & 6.37 \\
correlator with carrier smoothing & 7.12 & 9.38 \\
5. Differential single-epoch positioning using a & & \\
strobe correlator with carrier smoothing & 11.55 & 4.09 \\
6. EKF-based positioning with conventional & & 4.34 \\
receiver equivalent to VDLL & 9.51 & \\
7. VDLL & & \\
8. VDLL + strobe correlator (0.3,0.15) & 8.66 & \\
\hline
\end{tabular}



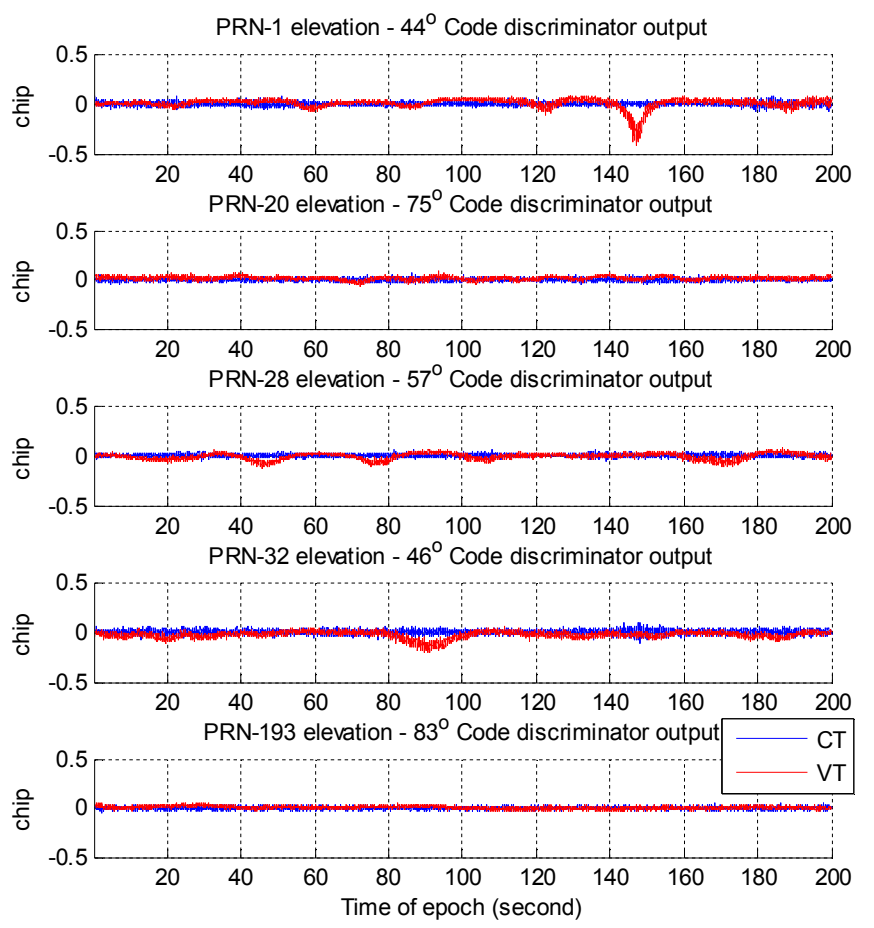

Figure 10: Code discriminator outputs using conventional and vector tracking in Toyosu, Tokyo

To predict the performance of vector tracking in a consumer receiver, a second experiment was conducted at the same site as the first using an iP-Solutions J-type front-end in place of the wide-bandwidth Fraunhofer front-end. The iP-Solutions J-type front-end has a precorrelation bandwidth of $4 \mathrm{MHz}$, an IF of $4.123968 \mathrm{MHz}$, and a sampling frequency of 16.367667 MHz. The NovAtel antenna was also replaced by a consumer-grade U-blox patch antenna. With this equipment, larger ranging errors in the presence of multipath interference can be expected. Figure 11 shows the code discriminator output and the range measurement noise variance of the channels with the strongest (PRN11) and weakest (PRN193) multipath interference. The range measurement noise variance is the variance of measurement innovation of the delta pseudo-ranges that mentioned earlier in the section of vector tracking. 

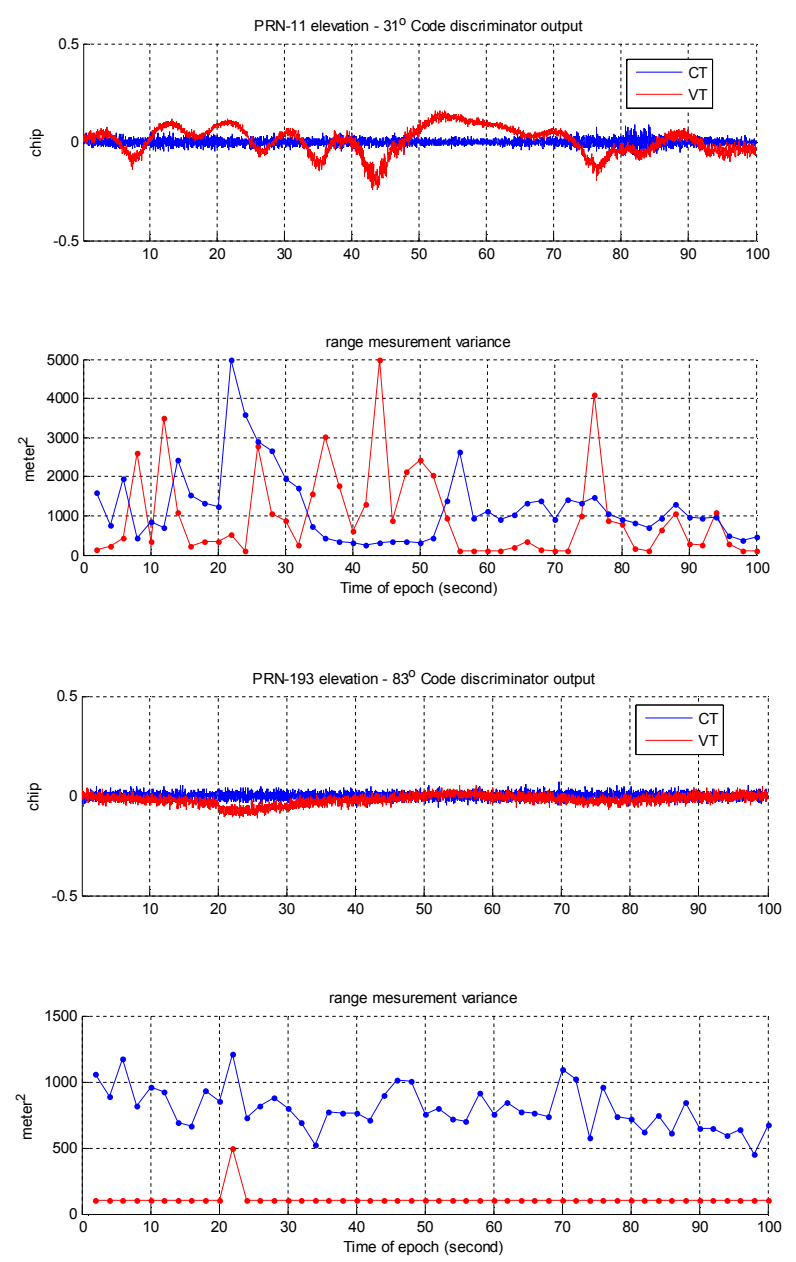

Figure 11: Examples of code discriminator outputs and range measurement variance of conventional and vector tracking in Toyosu, Tokyo

In the weak-multipath case, the code discriminator outputs of both conventional and vector tracking are similar as shown in the upper-column of lower Figure 11. However, the range-measurement noise variance is much smaller for vector tracking than for conventional tracking, which suggests that vector tracking is more stable. In the strong-multipath case, the effect of multipath on the code discriminator is quite strong as shown in upper-column of upper Figure 11. The received signal is assumed to be mainly sourced from multipath signals. It is interesting to note the lower-column of upper Figure 11; the range-measurement noise variance is higher for vector tracking than for conventional tracking in about half of the cases and correlates with the larger discriminator values. Therefore, the noise variance could potentially be used as a multipath detector. Note also that the vector-tracking algorithm automatically downweights measurements with high variances through its adaptive tuning algorithm.

The NLOS detection algorithm, described in the section of simulation, was assessed using 
the data collected with the iP-solutions front-end. The same settings were used. NLOS reception on PRN11 was detected between 44s and 46s. Figure 12 shows the positioning results using conventional tracking, vector tracking, vector tracking with the NLOS detection algorithm, and vector tracking with PRN 11 intentionally excluded. The standard vector-tracking algorithm produces a significantly more stable solution that either conventional tracking or vector tracking with PRN11 excluded throughout (leaving only 4 satellites tracked). With the NLOS detection algorithm, the vector-tracking solution is slightly more stable.
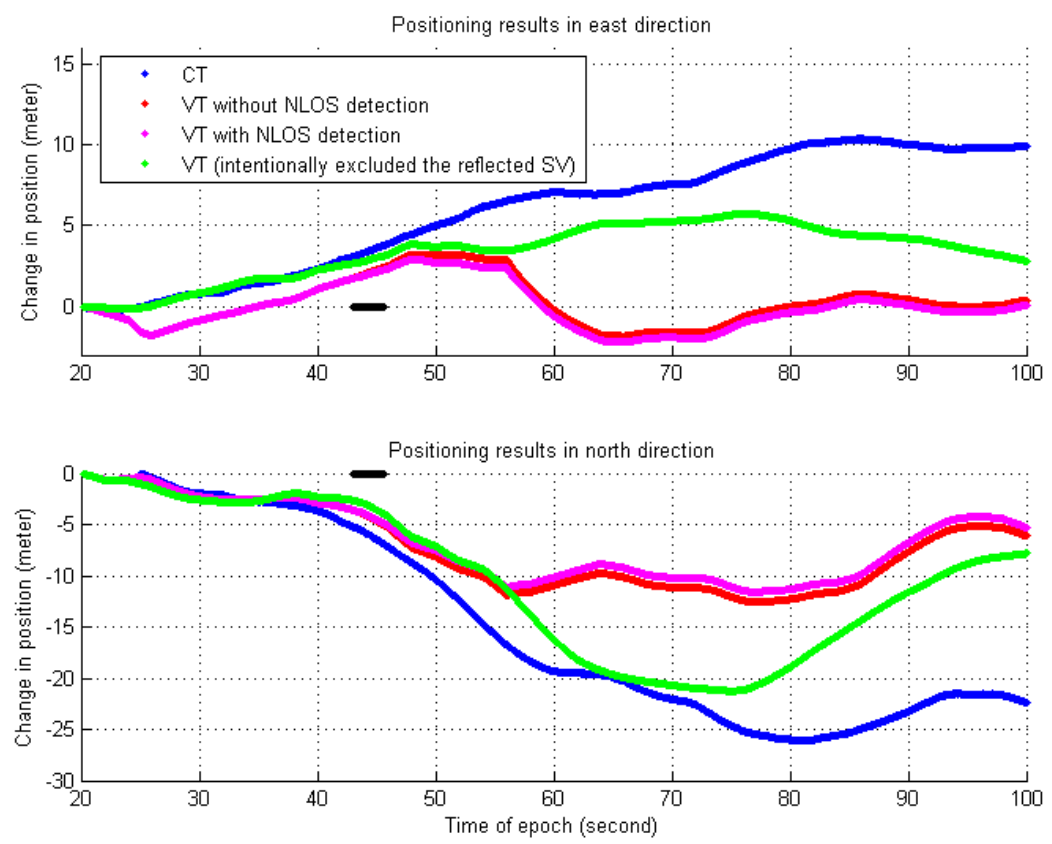

Figure 12: Horizontal positioning result using the iP-solutions front-end and four different receiver configurations in Toyosu, Tokyo

\section{Conclusions}

A new approach to mitigating multipath interference and NLOS reception, based on vector tracking, has been proposed and assessed. Vector tracking was originally developed for mitigating signal attenuation and interference for dynamic application. For multipath and NLOS mitigation, it has the advantage over many other techniques that it does not require additional hardware or a database.

Tests using GNSS signals recorded in dense urban environments have shown that vector tracking significantly improves GNSS positioning accuracy compared to an equivalent conventional receiver. A further improvement can be obtained by combining vector tracking 
with a correlator-based multipath mitigation technique, such as the strobe correlator.

Finally, a new technique for detecting NLOS reception in a vector-tracking architecture has been demonstrated. This enables NLOS signals to be excluded from the navigation solution, whilst retaining tracking lock using information from the other signals.

\section{Acknowledgments}

The first author acknowledges the Graduate Student Study Abroad Program (GSSAP) funded by National Science Council of Taiwan which provided financial support for his academic study in University College London. He also thanks Professor Marek Ziebart who provided him with the opportunity to work in the Space Geodesy and Navigation Laboratory (SGNL) at University College London. The authors appreciate all the useful suggestions and discussions from many members of SGNL.

The first author acknowledges the Summer Program funded by Japan Interchange Association which provided financial support for his academic study in Tokyo University of Marine Science and Technology. The authors also thank Dr. Taro Suzuki, a post-doctoral researcher at Tokyo University of Marine Science and Technology, for help of providing the open source GNSS software receiver and preparing the experiment in Tokyo.

\section{Reference}

Bhattacharyya S, Gebre-Egziabher D (2010) Integrity Analysis of Vector Tracking Architecture. Proc. ION-GNSS-2010, Institute of Navigation Portland, Oregon, September, pp 3152-3166

Bhuiyan MH, Lohan ES (2010) Advanced multipath mitigation techniques for satellite-based positioning applications, in Hindawi International Journal on Navigation and Observation, Volume 2010, Article ID 412393, 15 pages, doi:10.1155/2010/412393, http://www.hindawi.com/journals/ijno/2010/412393.html

Braasch MS (1996) Multipath Effects. In: B. W. Parkinson and J. J. Spilker (eds.) Global

Positioning System: Theory and Applications. Washington, DC: AIAA.

Brown RG, Hwang PYC (1996) Introduction to Random Signals and Applied Kalman Filtering. 3 edn. John Wiley \& Sons, New York, N.Y.

Copps EM, Geier GJ, Fidler WC, Grundy PA (1980) Optimal Processing of GPS Signals. NAVIGATION 27(3):171-182 
Dierendonck AJV, Fenton P, Ford T (1992) Theory and Performance of Narrow Correlator Spacing in GPS Receiver. Navigation: Journal of the Institute of Navigation, Vol. 39, No. 3, Fall 1992, pp. 265-284

Garin L, Diggelen Fv, Rousseau J-M (1996) Strobe \& Edge Correlator Multipath Mitigation for Code. In: Proc. ION-GPS-1996, Institute of Navigation Kansas City, MO, September, pp 657-664

Groves PD (2005) GPS Signal to Noise Measurement in Weak Signal and High Interference Environments. Navigation 52(2):83-92

Groves PD (2013) Principles of GNSS, Inertial, and Multi-Sensor Integrated Navigation Systems (GNSS Technology and Applications). 2nd edn. Artech House Publishers, Groves PD, Jiang Z (2013) Height Aiding, C/N $\mathrm{N}_{0}$ Weighting and Consistency Checking for GNSS NLOS and Multipath Mitigation in Urban Areas. Journal of Navigation 66 (5):653-659

Groves PD, Jiang Z, Rudi M, Strode P (2013) A Portfolio Approach to NLOS and Multipath Mitigation in Dense Urban Areas. In: Proc. ION-GNSS+-2013, Institute of Navigation Nashville, TN., September, pp. 3231 - 3247

Hsu LT (2013) Integration of Vector Tracking Loop and Multipath Mitigation Technique and Its Assessment. In: Proc. ION-GNSS-2013, Institute of Navigation Nashville, Tennessee, September, pp. 3263 - 3278

Hsu LT, Groves PD, Jan SS (2013) Assessment of the Multipath Mitigation Effect of Vector Tracking in an Urban Environment. In: Proc. of ION Pacific PNT, Honolulu, Hawaii, April, pp. 498 - 509

Hsu LT, Jan SS, Sun CC, Lin YC (2007) A New Algorithm for the Signal Cancellation of GIOVE-A L1B \& GPS L1 Signal. In: Proceedings of International Symposium on GPS/GNSS 2007, Persada Johor, Malaysia, November.

Jiang Z, Groves PD (2012) NLOS GPS signal detection using a dual-polarisation antenna. GPS Solutions

Jwo D-J, Tseng M-YCC-H, Cho T-S (2009) Adaptive and Nonlinear Kalman Filtering for GPS Navigation Processing. In: Moreno VM, Pigazo A (eds) Kalman Filter Recent Advances and Applications. InTech, Croatia.

Kanwal N, Hurskainen H, Nurmi J (2010) Vector tracking loop design for degraded signal environment. Ubiquitous Positioning Indoor Navigation and Location Based Service (UPINLBS), 2010 , vol., no., pp.1,4, 14-15 Oct. 2010, doi: 10.1109/UPINLBS.2010.5654015 
LaMance J, Collins P, Langley R (1996) Limiting Factors in Tropospheric Propagation Delay Error Modelling for GPS Airborne Navigation. In: Proc. of the ION-AM-1996, Cambridge, MA, June, pp 519-528

Lashley M, Bevly DM (2011) Comparison in the Performance of the Vector Delay/Frequency Lock Loop and Equivalent Scalar Tracking Loops in Dense Foliage and Urban Canyon. In: Proc. ION-GNSS-2011, Institute of Navigation Portland, Oregon, September, pp 1786-1803

Lashley M, Bevly DM, Hung JY (2009) Performance Analysis of Vector Tracking Algorithms for Weak GPS Signals in High Dynamics. Selected Topics in Signal Processing, IEEE Journal 3:661-673

Hernández-Pajares M,Juan J, Sanz J, Orus R， Garcia-Rigo A， Feltens J, Komjathy A, . Schaer SC, Krankowski A (2009) The IGS VTEC maps: a reliable source of ionospheric information since 1998. Journal of Geodesy 83 (3-4):263-275

McGraw GA, Young RSY, Reichenauer K, Stevens J, Ventrone F (2004) GPS Multipath Mitigation Assessment of Digital Beam Forming Antenna Technology in a JPALS Dual Frequency Smoothing Architecture. In: Proc. ION-NTM-2004, San Diego, CA, pp 561-572.

Mehra RK (1972) Approaches to Adaptive Filtering. IEEE Trans on Automatic Control 17 (5). doi:10.1109/TAC.1972.1100100

Misra P, Enge P (2011) Global Positioning System: Signals, Measurements, and Performance. Ganga-Jamuna Press, Lincoln, MA 01773

Mohammed AH, Schwarz KP (1999) Adaptive Kalman Filtering for INS/GPS. Journal of Geodesy 73:193-203

Pany T, Kaniuth R, Eissfeller B (2005) Deep Integration of Navigation Solution and Signal Processing. In: Proc. ION-ITM-2005, Long Beach, California, pp 1095 - 1102 Petovello MG, Lachapelle G (2006) Comparison of Vector-Based Software Receiver Implementations with Application to Ultra-Tight GPS/INS Integration. In: Proc. ION-GNSS-2006, Fort Worth, Texas, September, pp 1790-1799

Pratt AR (2004) Performance of Multi-path Mitigation Techniques at Low Signal to Noise Ratios. In: Proc ION-GNSS-2004, Long Beach, CA, September, pp 43-53

Saastamoinen J (1972) Contributions to the theory of atmospheric refraction. Bulletin Géodésique 105 (1):279-298

Spilker JJ (1996) Fundamentals of Signal Tracking Theory. In: Parkinson BW (ed) Global Positioning System: Theory and Application, vol 1. American Institute of Aeronautics, 
Washington DC,

Veitsel VA, Zhdanov AV, Zhodzishsky MI (1998) The Mitigation of Multipath Errors by Strobe Correlators in GPS/GLONASS Receivers. GPS Solutions 2 (2):8. doi: 10.1007/PL00000035

Ward PW, Betz JW, Hegarty CJ (2006a) Interference, Multipath, and Scintillation. In: E. D. Kaplan CJH (ed) Understanding GPS: Principles and Application. 2 edn. Artech House Publishers, Norwood, MA.

Ward PW, Betz JW, Hegarty CJ (2006b) Satellite Signal Acquisition, Tracking, and Data Demodulation. In: E. D. Kaplan CJH (ed) Understanding GPS: Principles and Application. 2 edn. Artech House Publishers, Norwood, MA.

Zhao S, Akos DM An Open Source GPS/GNSS Vector Tracking Loop - Implementation, Filter Tuning, and Results. In: Proc. ION-ITM-2011, San Diego, California, pp 1293-1305 